\title{
Use of an Ambulatory Patient Portal for Advance Care Planning Engagement
}

\author{
Adreanne Brungardt, MM, MT-BC, Andrea E. Daddato, MS, Bennett Parnes, MD, and \\ Hillary D. Lum, MD, PhD
}

Background: Primary care clinics need sustainable, population-based approaches to engage patients in advance care planning (ACP). Patient portal-based ACP tools may provide an option for patient engagement.

Objective: To increase ACP outcomes by engaging older adults through portal-based ACP tools, including an electronic Medical Durable Power of Attorney (MDPOA) form.

Methods: Geriatric clinic pilot of a multi-modal population-based outreach strategy for portal-based ACP tools. Outreach was to patients $(n=105)$ who were 65 years and older with an active portal account, no cognitive impairment, and no MDPOA on file. Patients received a motivational message via the portal and, if not read within 2 weeks, a mailed postcard about the portal-based ACP tools. Primary outcome was composite of any ACP action at 1-year including 1) new advance directive (AD) in the electronic health record, 2) use of portal-based ACP tools, or 3) documented ACP discussion with a provider.

Results: Sixty-five older adults read the electronic message at 12 months. Seventeen (16\%) engaged in at least 1 ACP action. Fourteen of 17 engaged by adding an AD to their record. More patients completed an AD on article or brought a previously completed AD to clinic, compared with choosing to complete an electronic MDPOA via the portal.

Conclusions: Brief motivational messages about ACP via a patient portal is feasible and may increase ACP outcomes for older adults in primary care. Future studies should evaluate population-based portal outreach strategies in combination with team-based workflows to enhance patient engagement in ACP. (J Am Board Fam Med 2019;32:925-930.)

Keywords: Advance Care Planning, Advance Directives, Doctor Patient Relations, Electronic Health Records, End of Life Care, Internet, Patient Participation, Primary Health Care, Quality Improvement, Workflow

Primary care practices have not yet routinely implemented practice models to engage all patients in advance care planning (ACP). ${ }^{1} \mathrm{ACP}$ is a process that supports adults in understanding and sharing

This article was externally peer reviewed.

Submitted 16 January 2019; revised 6 June 2019; accepted 21 June 2019.

From the Division of Geriatric Medicine, Department of Medicine, University of Colorado School of Medicine, Anschutz Medical Campus, Aurora, CO (AB, AED, BP, HDL); VA Eastern Colorado Geriatric Research Education and Clinical Center, Aurora, CO (HDL).

Funding: This initiative is funded by The Colorado Health Foundation with additional support from the $\mathrm{Na}$ tional Institute on Aging of the National Institutes of Health (K76AG054782) and the Colorado Clinical \& Translational Sciences Institute (CCTSI) with the Development and Informatics Service Center (DISC) grant support (NIH/ NCRR Colorado CTSI Grant Number UL1 RR025780) for use of REDCap-based data management. The content is personal values, life goals, and preferences regarding future medical care. ${ }^{2}$ Interventions to engage patients in ACP conversations are low risk and potentially high value for older patients. ${ }^{3-5}$ Recent studies of ACP interventions in primary care have found that communication guides for difficult conversations combined with changes in the electronic health record (EHR) increased ACP conversations

solely the responsibility of the authors and does not necessarily represent the official views of the National Institutes of Health. The sponsors had no role in the design, methods, or preparation of the manuscript. The views in this article are those of the authors and do not necessarily represent the views of the Department of Veterans Affairs.

Conflict of interest: none declared.

Corresponding author: Hillary D. Lum, MD, PhD, 12631 E. 17th Ave, Campus Mail Stop B179, Aurora, CO 80045

(E-mail: Hillary.Lum@ucdenver.edu). 
and documentation. ${ }^{6,7}$ Primary care-based models are also important because ACP metrics are increasingly included in value-based payment programs as a measure of quality care.

Despite the availability of evidence-based interventions for ACP and the association of ACP with improved quality at the end of life, more hospice use, and fewer in-hospital deaths, ${ }^{8,9}$ ACP engagement remains low in the United States. ${ }^{10,11}$ To address this challenge, Web-based tools have been proposed to improve access to ACP education at minimal cost compared with facilitator-based interventions. ${ }^{12}$ One novel method is use of EHR-based patient portals, a Web-based interface tethered to a patient's EHR. Use of patient portals has resulted in increased advance directives (ADs) in the EHR in a few studies. ${ }^{13-15}$ For example, Tieu et $\mathrm{al}^{16}$ conducted a study among primary care patients to test whether a motivational electronic message linked to an external ACP workbook, AD, and letter from the primary care provider (PCP), increased ADs in the EHR. The intervention group completed an $\mathrm{AD} 5.5 \%$ of the time compared with $2 \%$ in standard care. However, consistent with the majority of state laws, that portal-based ACP workflow required the necessary additional step of printing the $\mathrm{AD}$ for witness signatures or notarization, then bringing the $\mathrm{AD}$ to clinic.

This practice-based initiative aimed to pilot a population-based outreach using a portal-based electronic message and mailed postcard to motivate older adult engagement in ACP actions. This initiative used low-cost methods to increase awareness of available portal-based ACP tools including an electronic Medical Durable Power of Attorney (MDPOA) form, a patient-centered Web page, and online messaging to a centralized ACP Support Team. ${ }^{17}$ Building on studies using the portal to motivate patients to engage in ACP, this initiative combined a motivational message about ACP with ability to choose health care decision makers by completing an electronic MDPOA form within the portal in a single step.

\section{Methods}

\section{Design and Setting}

This clinical demonstration project was conducted in a primary care geriatric clinic in an academic health care system. The clinic includes 4 part-time PCPs and serves 525 patients, of whom approxi- mately $64 \%$ have a portal account. The intervention occurred from September 21, 2017 to November 27, 2017 with outcomes followed for 1 year. At baseline, $30 \%$ of patients had an MDPOA on file in the EHR. For comparison, a systematic review found $33 \%$ of US adults had an MDPOA form. ${ }^{18}$ The clinic's Patient Advisory Group provided input on the overall approach. The Colorado Multiple Institutional Review Board approved this as a quality improvement project. Patient consent was not required.

\section{Patient Portal Tools}

We implemented ACP tools into My Health Connection, the health system's patient portal integrated with the Epic EHR (Epic Systems, version 2017, Verona, WI). ${ }^{17}$ The ACP tools support an ACP process by 1) providing educational materials about ACP, including the importance of valuesbased discussions and information about living wills on a portal Web page, and 2) enabling legal appointment of a health-care decision maker through a MDPOA form. An electronic MDPOA is possible because Colorado state law only requires a patient signature to be valid; an electronic signature can be utilized; and the process is not accessible through proxy accounts. ${ }^{19}$ The current system-wide EHRbased process sends the electronic MDPOA forms for review by a centralized ACP Support Team, who then notify the PCP. The ACP Support Team includes a program coordinator and medical director who respond to patient on-line messages, phone calls, and review completed MDPOA forms, as detailed elsewhere. ${ }^{17}$ Although the ACP tools are accessible to any patient with a portal account, before this outreach, we have not conducted a marketing campaigns to raise awareness of the portalbased ACP tools.

\section{Participants}

Using a population-based approach and EHR report, we identified eligible patients for the intervention (Figure 1). Inclusion criteria included patients seen within the past 18 months, 65 years and older, and a portal account. Exclusion criteria included patients with an MDPOA in the EHR, cognitive impairment on the problem list (eg, dementia), or need for an interpreter. For this pilot, from 336 potentially eligible patients, 200 patients were manually reviewed for cognitive impairment and the presence of an MDPOA. 
Figure 1. Identification of Outreach Recipients. Electronic health record (EHR) report to identify English-speaking patients with a patient portal account. Two hundred patients were reviewed for eligibility as follows: (A) excluded for lack of Medical Durable Power of Attorney (MDPOA) document in EHR, and (B) excluded for cognitive impairment. *Thirteen patients had both an MDPOA and cognitive impairment. Eligible patients were sent portal outreach message. If portal message was unread at 14 days, patients were mailed an outreach postcard. Advance care planning (ACP) outcomes were collected at 1 year.

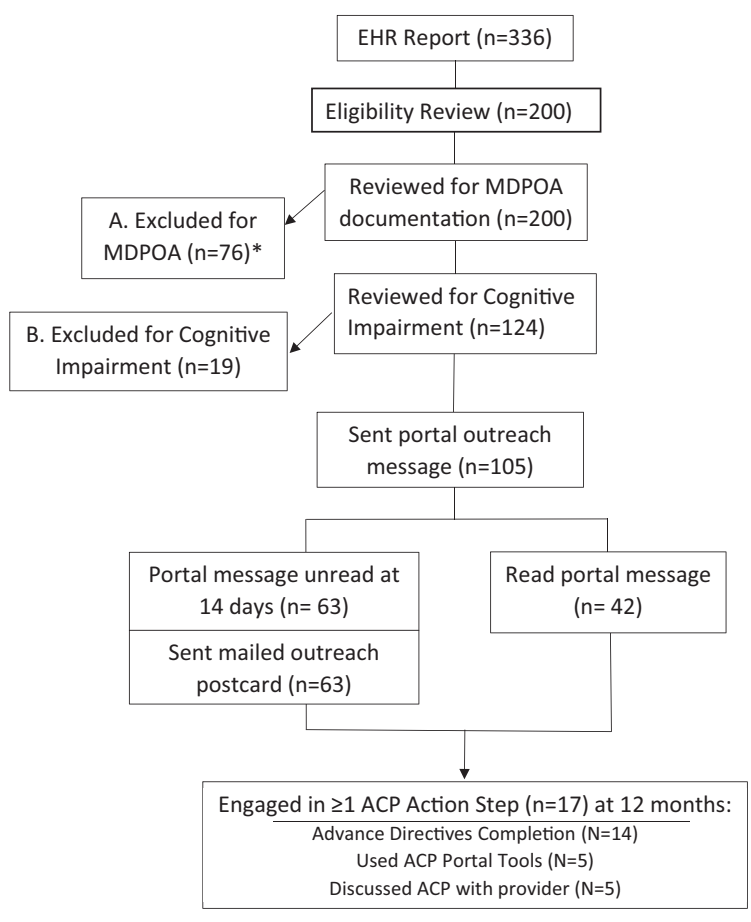

\section{Intervention}

Eligible patients were sent a motivational message about the importance of a health care decision maker (Appendix 1), their lack of an MDPOA in the EHR, instructions for accessing the portalbased ACP tools, and contact information for the centralized ACP Support Team for questions. Recognizing that some patients may not read the electronic message, within 2 weeks any patients who had not opened the message were mailed an informational postcard (Appendix 2) that also described the ACP tools.

\section{Evaluation}

The primary outcome was defined as a composite of any ACP action step including: 1) new $\mathrm{AD}$ in the
Table 1. Characteristics of Primary Care Patients Who Received an Advance Care Planning Portal-Based Outreach Initiative $(\mathbf{n}=105)$

\begin{tabular}{lc}
\hline Characteristic & $\mathrm{N}(\%)$ \\
\hline Age, Mean \pm SD & $77.7 \pm 5.9$ \\
Female & $67(64)$ \\
Patient registry & \\
$\quad$ Cancer & $41(39)$ \\
Diabetes & $18(17)$ \\
Chronic kidney disease & $15(14)$ \\
Congestive heart failure & $4(3.8)$ \\
Chronic obstructive pulmonary & $12(11)$ \\
$\quad$ disease & \\
Value-based payment program & \\
Comprehensive Primary Care Plus & $47(45)$ \\
Medicare Shared Savings Program & $46(44)$ \\
Advance care planning documentation & \\
$\quad$ at baseline & \\
Living will & $7(6.7)$ \\
MOST form & $61(58)$ \\
No ACP documents, but has provider & \\
$\quad$ documentation of orally appointed & \\
decision maker &
\end{tabular}

ACP, advance care planning; MOST, Medical Orders for Scope of Treatment; SD, standard deviation.

EHR, 2) use of portal-based ACP tools, or 3) documented ACP discussion with a provider, at 1 year. Any type of $\mathrm{AD}$ (ie, article-based MDPOA vs electronic MDPOA; living will; Medical Orders for Scope of Treatment form) was counted. Use of the portal-based ACP tools included completing the electronic MDPOA or sending a message through the portal. Outcomes were obtained via chart review at baseline, 14 days, 3 months, and 12 months. Data were securely stored in REDCap (Research Electronic Data Capture, Nashville, TN). As a process measure, the percentage of electronic messages read by patients at 14 days and 12 months was calculated.

\section{Results}

Of 200 patients reviewed for an existing MDPOA on file or presence of cognitive impairment, 105 patients $(53 \%)$ were appropriate for the intervention and received the motivational message through My Health Connection. Table 1 shows the demographics of patients who received the outreach.

Fourteen days after outreach, 42 of 105 patients had read the message. However, 63 patients had not read the message and were mailed the informa- 
tional postcard. At 1 year, 65 patients had read the message and 17 patients engaged in at least $1 \mathrm{ACP}$ action step (16\% engagement rate). Nine patients were under age 80 years and 8 patients were age 80 years and older. The timing of engagement varied: 2 patients took action within 14 days, 9 additional patients acted by 3 months, and 6 more by 12 months. Three patients engaged at multiple time points. Fourteen of 17 patients engaged by completing or adding an $\mathrm{AD}$ to the EHR, including 4 electronic MDPOAs, 10 article MDPOAs, 4 living wills, 2 Medical Orders for Scope of Treatment forms, and 1 CPR directive. Of the 17 patients who engaged in an ACP action, 5 patients used the portal-based ACP tools (4 electronic MDPOAs, 1 patient question to the ACP Support team). There were also 5 patients who had an in-person discussion with their PCP.

\section{Discussion}

Seventeen older adults engaged in ACP through an outreach process that raised awareness about portal-based ACP tools, including ability to complete an electronic MDPOA. More older adults chose to complete a new $\mathrm{AD}$ on article or bring to clinic a previously completed $\mathrm{AD}$ compared with choosing to complete an electronic MDPOA via the portal. Because patients engaged in different ACP actions, including $\mathrm{AD}$ completion, asking questions about $\mathrm{ACP}$, and having discussions with their PCP, this intervention may increase awareness about ACP as a process. Although these results describe the feasibility of a portal-based outreach process that may lead to ACP actions, further testing with a comparison group and mixed-methods evaluation is needed.

Our cohort has the oldest average age of studies demonstrating the feasibility of engaging patients in ACP via a patient portal. ${ }^{15,16}$ Over $62 \%$ of this older-adult cohort read the message within 1 year. This read rate exceeds rates of similar ACP interventions with younger cohorts. ${ }^{14,16}$ Despite the high read rate, the relatively lower use of the portal for ACP compared with article-based AD completion and in-person ACP discussions highlights the need for further evaluation of how and why older adults choose to engage in ACP, including through the patient portal. ${ }^{20}$ Our early work described perspectives of early adopters of the ACP tools. ${ }^{21}$ More than one-third of interviewees were age 60 years and older and had varying perspectives on usability of portal-based tools compared with article $\mathrm{ADs}$, however that analysis was not after an outreach process to raise awareness about the ACP tools.

This initiative has several limitations. This clinical demonstration project was conducted in a state that has an MDPOA form that does not require witnessing or notarization. The ACP tools and electronic MDPOA are currently only available in English, and incorporate use of an electronic signature, which may not be accepted in all states. Thus, these results are contextual and not generalizable. This outreach may have limited scalability to other practices. Bose-brill et $\mathrm{al}^{15}$ stratified outreach recipients into high and low portal users, with low portal users having less increase in ACP documentation. Others noted that patient acceptability of portal messaging may depend on how standardized portal use is by providers and staff. ${ }^{22}$ Thus, patients may not adopt or expect communication through their portal (regarding ACP or other topics) because portal usage is not the clinic norm.

Portal-based messages about ACP are feasible and may increase ACP outcomes for older adults in primary care. Future studies should investigate the combination of population-based portal outreach strategies with other in-person, team-based workflows to enhance patient engagement in ACP, and evaluate cost, sustainability, and other implementation outcomes.

This project would not have been possible without the organizational support of multiple UCHealth clinical leaders, including Drs. Jean Kutner (UCH Chief Medical Officer, coinvestigator), CT Lin (Chief Medical Information Officer), and the Lone Tree Seniors Clinic Patient Advisory Group. We also thank Josh Conrad, Leah Rusinek, and Sally Nietfeld for their work building and integrating the patient portal advance care planning tools, and Kate Sanfilippo for coordinating the My Health Connection Steering Committee.

To see this article online, please go to: http://jabfm.org/content/ 32/6/925. full.

\section{References}

1. Glaudemans JJ, Moll van Charante EP, Willems DL. Advance care planning in primary care, only for severely ill patients? A structured review. Fam Pract 2015;32:16-26.

2. Sudore RL, Lum HD, You JJ, et al. Defining advance care planning for adults: a consensus definition 
from a multidisciplinary Delphi panel. J Pain Symptom Manage 2017;53:821-832.e821.

3. Oczkowski SJ, Chung HO, Hanvey L, Mbuagbaw L, You JJ. Communication tools for end-of-life decision-making in ambulatory care settings: a systematic review and meta-analysis. PLoS One 2016;11: e0150671.

4. Lakin JR, Block SD, Billings JA, et al. Improving communication about serious illness in primary care: a review. JAMA Intern Med 2016;176:1380-1387.

5. Houben CHM, Spruit MA, Groenen MTJ, Wouters EF, Janssen DJ. Efficacy of advance care planning: a systematic review and meta-analysis. J Am Med Dir Assoc 2014;15:477-489.

6. Rose BL, Leung S, Gustin J, Childers J. Initiating advance care planning in primary care: a model for success. J Palliat Med 2019;22:427-431.

7. Lakin JR, Koritsanszky LA, Cunningham R, et al. A Systematic intervention to improve serious illness communication in primary care. Health Aff (Millwood) 2017;36:1258-1264.

8. Silveira MJ, Kim SY, Langa KM. Advance directives and outcomes of surrogate decision making before death. N Engl J Med 2010;362:1211-1218.

9. Pope TM. Legal briefing: Medicare coverage of advance care planning. J Clin Ethics 2015;26:361-367.

10. Grudzen CR, Buonocore P, Steinberg J, Ortiz JM, Richardson LD. Concordance of advance care plans with inpatient directives in the electronic medical record for older patients admitted from the emergency department. J Pain Symptom Manage 2016; 51:647-651.

11. Lamas D, Panariello N, Henrich N, et al. Advance care planning documentation in electronic health records: current challenges and recommendations for change. J Palliat Med 2018;21:522-528.

12. Chiarchiaro J, Arnold RM, White DB. Reengineering advance care planning to create scalable, patientand family-centered interventions. JAMA 2015;313: $1103-1104$.
13. Bajracharya AS, Crotty BH, Kowaloff HB, Safran C, Slack WV. Improving health care proxy documentation using a web-based interview through a patient portal. J Am Med Inform Assoc 2016;23:580-587.

14. Bose-Brill S, Feeney M, Prater L, Miles L, Corbett A, Koesters S. Validation of a novel electronic health record patient portal advance care planning delivery system. J Med Internet Res 2018;20(6):e208.

15. Bose-Brill S, Kretovics M, Ballenger T, et al. Testing of a tethered personal health record framework for early end-of-life discussions. Am J Manag Care 2016;22:e258-e263.

16. Tieu C, Chaudhry R, Schroeder DR, Bock FA, Hanson GJ, Tung EE. Utilization of patient electronic messaging to promote advance care planning in the primary care setting. Am J Hosp Palliat Care 2017; 34:665-670.

17. Lum HD, Brungardt A, Jordan SR, et al. Design and implementation of patient portal-based advance care planning tools. J Pain Symptom Manage 2019;57: 112-117.e2.

18. Yadav KN, Gabler NB, Cooney E, et al. Approximately one in three US adults completes any type of advance directive for end-of-life care. Health Aff (Millwood) 2017;36:1244-1251.

19. Latulipe C, Quandt SA, Melius KA, et al. Insights into older adult patient concerns around the caregiver proxy portal use: qualitative interview study. J Med Internet Res 2018;20(11):e10524.

20. Sakaguchi-Tang DK, Bosold AL, Choi YK, Turner AM. Patient portal use and experience among older adults: systematic review. JMIR Med Inform 2017; 5(4):e38.

21. Jordan SR, Brungardt A, Phimphasone-Brady P, Lum HD. Patient perspectives on advance care planning via a patient portal. Am J Hosp Palliat Care 2019;36:682-687.

22. Wolcott V, Agarwal R, Nelson DA. Is provider secure messaging associated with patient messaging behavior? Evidence from the US Army. J Med Internet Res 2017;19:1-1. 


\section{Appendix 1. Motivational Electronic Message}

Dear [Patient Name]

Thank you for partnering with us for your care. We are writing to let you know some information about your My Health Connection (MHC) account.

In Colorado, no 1 is approved to make medical decisions for another adult if they were too sick to make those decisions for themselves. This means not spouses, adult children, nor other family members. Because of this, it is very important to name a person who would speak for your beliefs based on your wishes.

As a UCHealth patient, you can choose a decision maker by filling out a legal form in your MHC account. This form is called a Medical Durable Power of Attorney (MDPOA). It is easy to complete and can be filled out on-line. It is changed right then in your electronic health record. Once filled out, it is valid anywhere in Colorado.

We do not have a MDPOA on file for you.

To learn more and to fill out a MDPOA:

1. Go to your MHC account using a desktop computer (not the mobile app).

2. Once there, click on "My Record" on the top banner.

3. Then, click on "Advance Care Planning" and click on the link "Identify your health care decision maker and complete a Medical Durable Power of Attorney form."
It is important to help us keep your medical record up to date. Please call us or bring a copy to your next visit if you already have any of these:

- a living will

- MDPOA

- medical orders for scope of treatment

- CPR directive

If you have any questions, please call us at 303-7243141. We look forward to working with you.

Sincerely,

Advance Care Planning Support Team on behalf of [Patient Provider]

\section{Appendix 2. Postcard Images}

Who would speak for you if you could not speak for yourself? UCHealth

Health Promotion Services Mail Stop B180, Academic Office One 12631 E 17th Ave Aurora, CO 80045 .

Take charge of your future medical care.

UCHealth wants to ensure your preferences are known. Telling us who you trust is the first step.

A Colorado Medical Durable Power of Attorney form lets you legally choose a person to make health care decisions on your behalf in the event you are unable to make decisions for yourself.

Quick. Free. Important. Fill out a form.

Visit Advance Care Planning at: www.uchealth. org/myhealthconnection Advance Care Planning Support Team 303.724.3141. 